\title{
The passive stretching, massage, and muscle energy technique effects on range of motion, strength, and pressure pain threshold in musculoskeletal neck pain of young adults
}

\author{
Hye Mi Jeong ${ }^{a}$, Jae-Hoon Shim ${ }^{a}$, Hye Rim Suh ${ }^{b}$ \\ ${ }^{a}$ Department of Physical Therapy, Division of Health Science, Baekseok University, Cheonan, Republic of Korea \\ ${ }^{\mathrm{b}}$ Department of Physiology, Neuroscience Research Institute, Korea University College of Medicine, Seoul, Republic of Korea
}

Objective: Musculoskeletal neck pain have many symptoms which include decreased range of motion (ROM) and muscle strength, and increased pain. However, the management methods are controversial. The purpose of this study was to examine the effects of three interventions on ROM, strength, and pressure pain threshold (PPT) with musculoskeletal neck pain.

Design: Pretest-posttest design.

Methods: Thirty subjects participated in this experiment. They were randomly assigned to thefollowing groups: passive stretching (PS) group $(n=10)$, massage (MASS) group $(n=10)$, and muscle energy technique (MET) group ( $n=10)$. The treatment were applied bilaterally on the upper trapezius. The PS was applied 3 times for 30 seconds each time. The MASS was applied using two different techniques for 2 minutes per technique. For MET, the subjects performed 2 sets of 3 repetitions of isometric resistance exercise that was maintained for 10 seconds, followed by 10 seconds of rest. ROM, strength, and PPT parameters were measured after intervention.

Results: In the MASS group, there was a significant improvement in all outcomes except for muscle strength $(p<0.05)$. In the MET group, ROM and strength significantly improved compared to the pre-treatment results $(p<0.05)$. As result of measuring the amount of change in each group, there was a significant difference in ROM (flexion) in the PS group compared with the MASS and MET group, a significant difference in strength in the MET group compared with the PS and MASS groups, and a significant difference in PPT in the MASS groups compared with the PS and MET groups $(p<0.05)$.

Conclusions: This study showed that PS, MASS, and MET are effective methods for improving ROM, strength, and PPT for musculoskeletal neck pain. Therefore, various therapeutic interventions for improving ROM, strength, and pain are suggested.

Key Words: Massage, Muscle stretching exercises, Musculoskeletal pain

\section{Introduction}

Modern musculoskeletal pain is often generated by the increased use of computers and sustained seated posture [1]. Studies have shown that neck pain in $67 \%$ of the total population has been experienced due to overuse of muscles in the scapular and neck region amongst those with musculoskeletal disorders in modern society [2]. Most cases of neck pain are not due to an anatomical disorder, but instead are associated with a functional abnormality. Neck pain can cause muscle pain, fatigue, and migraines. It can also trigger radiating pain, muscular weakness, and atrophy, as radiculopathy associated with restricted range of motion (ROM) may develop due to of neck stiffness and pain [3]. People with chronic neck pain experience difficulties with performing their activities of daily living [4]. Thus, early effective

Received: 9 November, 2017 Revised: 15 December, 2017 Accepted: 15 December, 2017

Corresponding author: Hye Rim Suh

Department of Physiology, Korea University College of Medicine, 73 Inchon-ro, Seongbuk-gu, Seoul 02841, Republic of Korea

Tel: 82-2-2286-1282 Fax: 82-2-927-1036 E-mail: hyerimsuh@gmail.com

(c) This is an Open-Access article distributed under the terms of the Creative Commons Attribution Non-Commercial License (http://creativecommons.org/licenses/ by-nc/4.0) which permits unrestricted non-commercial use, distribution, and reproduction in any medium, provided the original work is properly cited.

Copyright @ 2017 Korean Academy of Physical Therapy Rehabilitation Science 
management of acute neck pain is necessary prior to the generation of chronic neck pain.

Recently, various managements have been applied to musculoskeletal pain, including the use of oral medications and more invasive treatment, such as drug injections. However, oral medications and drug injections have other effects, which may include muscle relaxation or the induction of non-protective movement due to hyper-inhibition of pain transmission. These treatments may also have other side effects, including digestive problems [5]. The application of non-invasive treatment for musculoskeletal pain includes the use of stretching, massage, and muscle energy technique (MET). The first technique includes stretching which has an effect on restoring the original muscle length along with recovering ROM. It also increases the muscle torque and exhibits an analgesic effect by increasing the pain threshold [6]. The second technique is massage, which is applied to soft tissues and can contribute to alteration of blood flow, blood pressure, and skin temperature using the hands, a vibrator, and a roller [7,8]. The third technique is MET followed by relaxation and passive stretching (PS). The effects of MET includes increase strength and lengthening, as well as improved circulation [9]. The effects of these management techniques on muscular pain or movement disorders in general are well known; however, the effects of their application on musculoskeletal neck diseases are not well known. It is important to investigate these techniques in modern society due to the increases in neck/shoulder tenderness and pain caused by continuous static working positions other than general muscle pain induced by exercise or injury [10].

Thus, the purpose of this study was to compare the effects of stretching, massage, and MET on the ROM, strength, and pain of the neck. We also propose a variety of therapeutic interventions to improve ROM, muscle strength, and pain associated with neck disorders.

Table 1. General characteristics of the participants $\quad(\mathrm{N}=30)$

\begin{tabular}{lrcc}
\hline Characteristic & Male $(\mathrm{n}=13)$ & Female $(\mathrm{n}=17)$ & Total \\
\hline Age $(\mathrm{yr})$ & $22.4(1.21)$ & $20.8(2.34)$ & $21.5(1.52)$ \\
Height $(\mathrm{cm})$ & $173.7(6.42)$ & $161.8(5.51)$ & $167.4(8.71)$ \\
Weight $(\mathrm{kg})$ & $69.4(9.11)$ & $55.6(5.72)$ & $61.6(10.04)$ \\
\hline
\end{tabular}

Values are presented as mean (SD).

\section{Methods}

\section{Subjects}

Thirty participants (13 male, 17 female) with the following demographics participated in the study (Table 1). The participants of Baekseok University located in Cheonan, Korea, provided written informed consent for the experimental procedure. All participants provided their informed consent. This study included participants who had forward neck postures with located in anterior position of cervical spine. None of the participants had any known history of neurological disorders or congenital anomalies.

\section{Experimental procedures}

The subjects comfortably sat in a resting position and maintained the position of their scapulae against the back of the chair for passive stretching, massage, and MET (Figure 1). The experimenter performed the stretches by creating a motion involving lateral flexion and rotation of the neck on both sides without generation of pain. The stretching motion was applied 3 times for 30 seconds each time [11]. The pick-
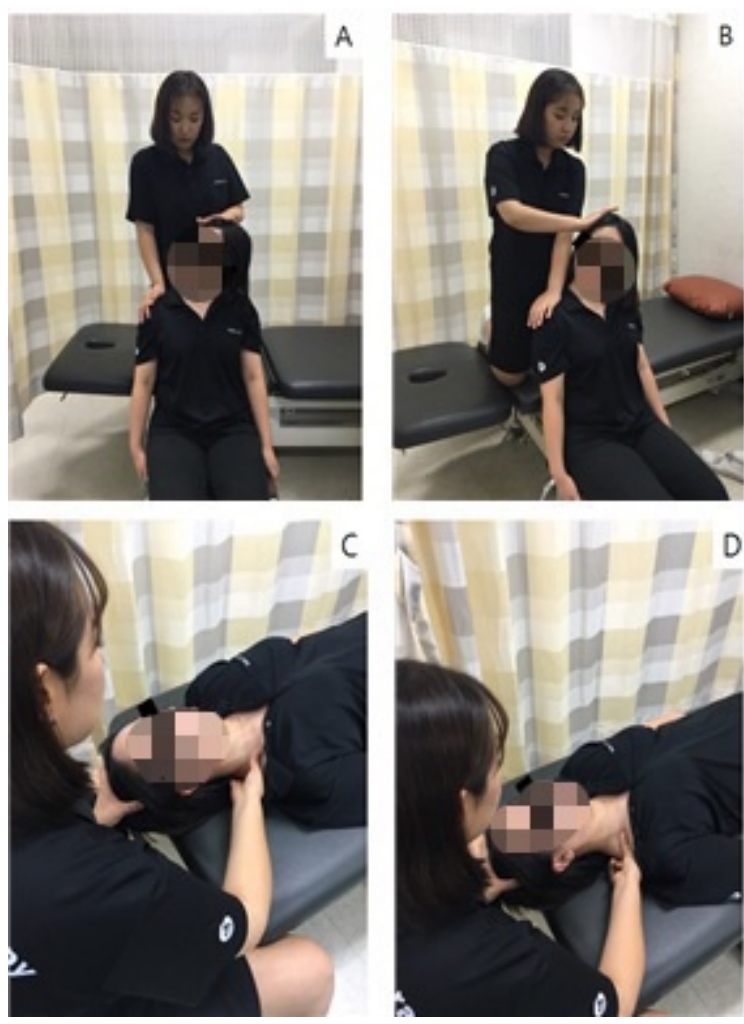

Figure 1. Application of assessments. (A) Passive stretching. (B) Muscle energy technique. (C) Stripping method of massage. (D) Picking-up-with-palpation method of massage. 
ing-up-with-palpation methods of massage were applied to the upper trapezius muscles bilaterally, with equal time spent on both sides. The striping method involves applying pressure while moving along from the origin to the insertion of the muscle with parallel strokes, depending on the direction of the muscle fibers. The picking-up-with-palpation method involves the use of the sides of the thumb, index finger and middle finger to hold the muscle tissue. The fingers were applied to provide a firm surface of soft tissue for the muscle relaxation to press and promote. The application time of the massage was 2 minutes per technique [12]. The subjects were instructed to bend and twist their heads comfortably in a seated position, as well as to perform PS exercises. Then, the examiner placed on the shoulders of the subjects with the opposite hand on the head to resist isometric contraction for MET. One set of a voluntary, gentle isometric resistance exercise was maintained for $10 \mathrm{sec}-$ onds, followed by 10 seconds of rest. This was repeated 3 times. Each subject performed 2 sets and had a rest period of 10 seconds between the sets [9].

\section{Outcome measurement}

The protocol consisted of measuring the ROM, strength, and pressure pain threshold (PPT) of the trapezius muscle before and after PS, MASS, and MET while assuming a relaxed seated position and maintaining $90^{\circ}$ flexion of the hip and knee joints in the back of a chair.

The joint ROM of the neck was measured using a ROM analyzer (CROM Deluxe; Performance Attainment Associates, Lindstrom, MN, USA) before and after treatment. The measurements were conducted while the subjects performed bending and rotation movements on the left and right sides of the neck. Subjects were asked to look forward in a comfortable sitting position for the performance of the measurements. The initial position of each movement was set an angle of 0 degrees. Test-retest reliability of the CROM was reported to have an intra-class correlation (ICC) coefficient ranging between 0.89 and 0.98 [13]. The Muscle tester (JTECH Inc., Midvale, UT, USA) was used to measure muscle strength of the predominant upper trapezius muscle in a comfortable chair. When the examiner applied resistance, they instructed the subjects to elevate their scapulae. The resistance was applied 3 times per subject, each of whom rested for 10 seconds after holding the elevated position for 5 seconds. This tool has intra-rater $(\mathrm{ICC}=0.992)$ and inter-rater reliabilities (ICC $=0.949)$ [14]. The pressure pain threshold was measured using an algometer (JTECH Inc.) in a comfortable sitting position. The algometer was applied to the trigger point area, which was located at the midpoint between the spinous process of $\mathrm{C} 7$ and the acromion, with the device applied in perpendicular orientation to the surface of the skin. Compression of the area was performed slowly to elicit a reaction when pain was felt. The subjects were asked to say "pain" when they experienced an increase in pain intensity and the examiner stopped the compression. The average value of 3 repetitive measurements with an interval of 30 to 60 seconds (expressed as $\mathrm{kg} / \mathrm{cm}^{2}$ ) was collected for data analysis of the PPT. The method showed an inter-rater reliability ranging from 0.68 to 0.79 and an intra-rater reliability ranging from 0.71 to 0.92 when applied to the upper trapezius [15].

\section{Statistical analysis}

All data were analyzed with IBM SPSS Statistics ver. 18.0 (IBM Co., Armonk, NY, USA). For information pertaining to the general characteristics of the subjects, descriptive statistics were expressed as averages and standard deviations. For the analysis of the before-and-after results of each intervention, a paired t-test was performed to determine their effects on ROM, strength, and PPT. A one-way repeated ANOVA was used to determine the differences in ROM, muscle strength, and pain within the group. The Bonferroni method was used for the post-hoc analysis and the significance level was set at $\alpha=0.05$.

\section{Results}

\section{Before and after application in each group}

The comparison of ROM, muscle strength, and PPT in the PS, MASS, and MET groups before and after the treatment in each group is described in Table 2. The PS group was not significantly different in muscle strength, however, the ROM in this group increased from $32.50 \pm 10.68$ to $44.00 \pm$ 12.65 (lateral bending) and from $43.00 \pm 8.88$ to $52.20 \pm 10.78$ (rotation) $(p<0.05)$. In the MASS group, there was a significant difference in all outcomes after treatment, except for muscle strength. Lateral bending increased from $32.00 \pm 9.19$ to $38.20 \pm 7.32$ and the pain threshold showed the greatest increase from $18.60 \pm 15.53$ to $23.10 \pm 16.15(p<0.05)$. Furthermore, in the MET group, there was a significant difference in ROM from $30.20 \pm 4.13$ to $37.60 \pm 4.35$ and the muscle strength and strength of the muscles increased from $34.70 \pm$ 14.73 to $38.00 \pm 14.34(p<0.05)$, but not in PPT $(p<0.05)$. 
Table 2. Comparison between pre- and post-assessment in PS, MASS, and MET

$(\mathrm{N}=30)$

\begin{tabular}{|c|c|c|c|}
\hline Group & Pre-test & Post-test & $\mathrm{t}(p)$ \\
\hline \multicolumn{4}{|l|}{ PS $(n=10)$} \\
\hline Lateral flexion ROM $\left(^{\circ}\right)$ & $32.50(10.68)$ & $44.00(12.65)^{\mathrm{a}}$ & $-6.87(<0.001)$ \\
\hline Rotation ROM $\left(^{\circ}\right)$ & $43.00(8.88)$ & $52.20(10.78)^{\mathrm{a}}$ & $-6.55(<0.001)$ \\
\hline Strength $(\mathrm{kg})$ & $28.70(14.14)$ & $30.20(12.66)$ & $-2.04(0.07)$ \\
\hline Pressure pain threshold (kg) & $16.20(0.59)$ & $17.90(10.30)^{\mathrm{a}}$ & $-2.38(0.04)$ \\
\hline \multicolumn{4}{|l|}{ MASS $(n=10)$} \\
\hline Lateral flexion $\operatorname{ROM}\left({ }^{\circ}\right)$ & $32.00(9.19)$ & $38.20(7.32)^{b}$ & $-5.38(<0.001)$ \\
\hline Rotation $\operatorname{ROM}\left({ }^{\circ}\right)$ & $51.50(10.01)$ & $59.00(11.97)^{\mathrm{b}}$ & $-4.03(<0.001)$ \\
\hline Strength $(\mathrm{kg})$ & $30.80(11.75)$ & $30.30(12.09)$ & $1.25(0.24)$ \\
\hline Pressure pain threshold $(\mathrm{kg})$ & $18.60(15.53)$ & $23.10(16.15)^{b}$ & $-8.29(<0.001)$ \\
\hline \multicolumn{4}{|l|}{$\operatorname{MET}(n=10)$} \\
\hline Lateral flexion $\operatorname{ROM}\left({ }^{\circ}\right)$ & $30.20(4.13)$ & $37.60(4.35)^{\mathrm{c}}$ & $-5.84(<0.001)$ \\
\hline Rotation $\operatorname{ROM}\left(^{\circ}\right)$ & $39.00(14.10)$ & $52.00(15.13)^{\mathrm{c}}$ & $-3.34(0.01)$ \\
\hline Strength $(\mathrm{kg})$ & $34.70(14.73)$ & $38.00(14.34)^{\mathrm{c}}$ & $-6.99(<0.001)$ \\
\hline Pressure pain threshold (kg) & $19.70(9.43)$ & $21.00(9.56)$ & $-1.71(0.12)$ \\
\hline
\end{tabular}

Values are presented as mean (SD).

PS: passive stretching, MASS: massage, MET: muscle energy technique, ROM: range of motion.

${ }^{a} \mathrm{~A}$ significant difference from the pre-test value in PS group $(p<0.05)$. ${ }^{\mathrm{b}} \mathrm{A}$ significant difference from the pre-test value in massage group $(p<0.05) .{ }^{\mathrm{C}} \mathrm{A}$ significant difference from the pre-test value in MET group $(p<0.05)$.

Table 3. Comparison of variation in before and after measurement in PS, MASS, and MET groups

$(\mathrm{N}=30)$

\begin{tabular}{lcccc}
\hline \multicolumn{1}{c}{ Variable } & PS $(\mathrm{n}=10)$ & MASS $(\mathrm{n}=10)$ & MET $(\mathrm{n}=10)$ & $\mathrm{F}(p)$ \\
\hline Lateral flexion $\operatorname{ROM}^{\circ}\left(^{\circ}\right)$ & $11.50(5.30)^{\mathrm{a}}$ & $6.20(3.65)$ & $7.40(4.01)$ & $4.04(0.03)$ \\
Rotation ROM $\left(^{\circ}\right)$ & $9.20(4.44)$ & $7.50(5.89)$ & $13.00(12.29)$ & $1.16(0.33)$ \\
Strength $(\mathrm{kg})$ & $1.50(2.32)$ & $-0.50(1.27)$ & $4.50(1.84)^{\mathrm{a}}$ & $18.29(<0.001)$ \\
Pressure pain threshold $(\mathrm{kg})$ & $1.70(2.26)$ & $4.90(1.79)^{\mathrm{a}}$ & $1.30(2.41)$ & $8.27(<0.001)$ \\
\hline
\end{tabular}

Values are presented as mean (SD).

PS: passive stretching, MASS: massage, MET: muscle energy technique, ROM: range of motion.

${ }^{a}$ A significant difference from change value of before and after test in PS, massage, and MET group in lateral flexion ROM, strength, and pressure pain threshold $(p<0.05)$.

\section{Comparison of pre and post application between groups}

The comparison of the change in values before and after the application of treatment between each group was significantly different in lateral flexion ROM, strength, and pressure pain thresholds of the PS, MET, and MASS groups (Table 3) There was a significant difference among all three groups except in the values associated with ROM (rotation) $(p<0.05)$.

\section{Discussion}

In this study, we investigated the effects of three interventions (PS, MET, and MASS) that can increase ROM of the neck, increase muscle strength of the trapezius, and im- prove pain management. There was a significant increase in ROM and decrease in pain with the application of PS, and a significant increase in ROM and muscle strength when MET was applied. Also, when massage was applied, there was a significant difference in ROM and PPT, but not in muscle strength.

PS reduced the stiffness of the muscle-tendon unit as adaptations of the proprioceptive system occurred [16]. The use of static stretching at the end of painful muscles has been reported to increase ROM and reduce pain by inducing pain tolerance [17]. The increase in ROM is closely related to the increase in the pain threshold. In the present study, it is considered that the rotational ROM and left and right side bending ROM increased due to the decrease in pain. PS has been mentioned in many previous studies as having an effect on 
muscle strength reduction. The force reduction occurs as a result of a change in the length-tension relationship, reduction in neural drive, and perception of joint position $[18,19]$. In our current experiment, this did not have a significant effect on the improvement of muscle strength during manual stretching.

Massage lead to the secretion of hormones, causing stimulation of mechanoreceptors, and eliminated the accumulation of lactate and inflammatory cytokines as a result of increased lymph and blood circulation, leading to pain relief [20]. The activity of intrafusal and extrafusal fibers activated in the shortened position of painful muscles and massage reduced the muscle spindle activity and muscle tension associated with pain [21]. The cytokines of tumor necrosis factor- $\alpha$ and interleukin- 6 generate an inflammatory state and stimulate the nociceptive fibers, thus innervating them [22]. The cytokines are inhibited by massage in delayed onset muscle soreness of the quadriceps, which has been demonstrated on biopsy [23]. These results suggest that increased ROM and reduced pain are showed by decreased inflammation in our experiment.

MET is applied to target muscles as they are lengthening and strengthening, leading to decreased local edema and increased lymphatic fluid movement. MET has been shown to improve in strength and pain in chronic lateral epicondylitis [24]. MET inhibits the motor activation of Golgi tendon organs and isometric contraction causes lengthening of viscoelastic and plastic changes in myofascial connective tissue. Active exercise affects total muscles and relates to more specific movement with surrounding connective tissue [25]. Additionally, MET increases regional cervical ROM during the motions of rotation and lateral bending, as well as lumbar extension when applied to the thoracic and sacroiliac regions [26]. Active exercise affects total muscles and relates to more specific movement with surrounding connective tissue [25]. In our study, cervical ROM and strength in the upper region of the shoulder improved more with MET than with PS and massage; this might be due to the inhibition of Golgi tendon organs and length changes in connective tissue due to the application of active movement.

One limitation of this study was that there were a limited number of subjects in the experiment group and other measurements. In addition, it was difficult to demonstrate the major effects of the three interventions because they were applied for a short period of time. Also, it is difficult to know how long the effects had been maintained after the intervention because of no follow-up study. Future studies should test the generalizability by including those who work in the office or perform strenuous work.

In this study, we measured ROM (lateral bending and rotation), muscle strength, and pain before and after each intervention. Our results showed that PS was effective for increasing ROM, and massage was an appropriate treatment for pain relief. Also, MET was more effective for improving muscle strength. Furthermore, the results of this study will help to produce positive results for clinicians responsible for the selection of therapeutic interventions designed to prevent and treat chronic neck pain in the clinic.

\section{Conflict of Interest}

The authors declared no potential conflicts of interest with respect to the authorship and/or publication of this article.

\section{References}

1. Falla D. Unravelling the complexity of muscle impairment in chronic neck pain. Man Ther 2004;9:125-33.

2. Cheng CH, Wang JL, Lin JJ, Wang SF, Lin KH. Position accuracy and electromyographic responses during head reposition in young adults with chronic neck pain. J Electromyogr Kinesiol 2010;20:1014-20.

3. Rezasoltani A, Ahmadipoor A, Khademi-Kalantari K, Javanshir $\mathrm{K}$. The sign of unilateral neck semispinalis capitis muscle atrophy in patients with chronic non-specific neck pain. J Back Musculoskelet Rehabil 2012;25:67-72.

4. Harvold M, MacLeod C, Vaegter HB. Attentional avoidance is associated with increased pain sensitivity in patients with chronic posttraumatic pain and comorbid posttraumatic stress. Clin J Pain 2018;34:22-9.

5. Hunold KM, Esserman DA, Isaacs CG, Dickey RM, Pereira GF, Fillingim RB, et al. Side effects from oral opioids in older adults during the first week of treatment for acute musculoskeletal pain. Acad Emerg Med 2013;20:872-9.

6. Herman SL, Smith DT. Four-week dynamic stretching warm-up intervention elicits longer-term performance benefits. J Strength Cond Res 2008;22:1286-97.

7. Wang JS. Therapeutic effects of massage and electrotherapy on muscle tone, stiffness and muscle contraction following gastrocnemius muscle fatigue. J Phys Ther Sci 2017;29:144-7.

8. Guo J, Li L, Gong Y, Zhu R, Xu J, Zou J, et al. Massage alleviates delayed onset muscle soreness after strenuous exercise: a systematic review and meta-analysis. Front Physiol 2017;8:747.

9. Goodridge JP. Muscle energy technique: definition, explanation, methods of procedure. J Am Osteopath Assoc 1981;81:249-54.

10. Brandt M, Sundstrup E, Jakobsen MD, Jay K, Colado JC, Wang $\mathrm{Y}$, et al. Association between neck/shoulder pain and trapezius muscle tenderness in office workers. Pain Res Treat 2014;2014: 352735. 
11. Decoster LC, Cleland J, Altieri C, Russell P. The effects of hamstring stretching on range of motion: a systematic literature review. J Orthop Sports Phys Ther 2005;35:377-87.

12. Clay JH, Pounds DM. Basic clinical massage therapy: integrating anatomy and treatment. 2nd ed. Philadelphia: Lippincott Williams \& Wilkins; 2008.

13. Audette I, Dumas JP, Côté JN, De Serres SJ. Validity and between-day reliability of the cervical range of motion (CROM) device. J Orthop Sports Phys Ther 2010;40:318-23.

14. Kim SG, Lim DH, Cho YH. Analysis of the reliability of the make test in young adults by using a hand-held dynamometer. $\mathrm{J}$ Phys Ther Sci 2016;28:2238-40.

15. Takala EP. Pressure pain threshold on upper trapezius and levator scapulae muscles. Repeatability and relation to subjective symptoms in a working population. Scand J Rehabil Med 1990;22:63-8.

16. Apostolopoulos N, Metsios GS, Flouris AD, Koutedakis Y, Wyon MA. The relevance of stretch intensity and position-a systematic review. Front Psychol 2015;6:1128.

17. Blazevich AJ, Cannavan D, Waugh CM, Miller SC, Thorlund JB, Aagaard P, et al. Range of motion, neuromechanical, and architectural adaptations to plantar flexor stretch training in humans. J Appl Physiol (1985) 2014;117:452-62.

18. Trajano GS, Seitz L, Nosaka K, Blazevich AJ. Contribution of central vs. peripheral factors to the force loss induced by passive stretch of the human plantar flexors. J Appl Physiol (1985) 2013; 115:212-8.

19. Streepey JW, Mock MJ, Riskowski JL, Vanwye WR, Vitvitskiy
BM, Mikesky AE. Effects of quadriceps and hamstrings proprioceptive neuromuscular facilitation stretching on knee movement sensation. J Strength Cond Res 2010;24:1037-42.

20. Kelencz CA, Tarini VA, Amorim CF. Trapezius upper portion trigger points treatment purpose in positional release therapy with electromyographic analysis. N Am J Med Sci 2011;3:451-5.

21. Alagesan J, Shah US. Effect of positional release therapy and taping on unilateral upper trapezius tender points - randomized control trial. Int J Health Pharm Sci 2012;1:13-7.

22. LeGrand A, Fermor B, Fink C, Pisetsky DS, Weinberg JB, Vail $\mathrm{TP}$, et al. Interleukin-1, tumor necrosis factor alpha, and interleukin-17 synergistically up-regulate nitric oxide and prostaglandin E2 production in explants of human osteoarthritic knee menisci. Arthritis Rheum 2001;44:2078-83.

23. Crane JD, Ogborn DI, Cupido C, Melov S, Hubbard A, Bourgeois JM, et al. Massage therapy attenuates inflammatory signaling after exercise-induced muscle damage. Sci Transl Med 2012;4:119ra13.

24. Küçükşen S, Yilmaz H, Sallı A, Uğurlu H. Muscle energy technique versus corticosteroid injection for management of chronic lateral epicondylitis: randomized controlled trial with 1-year follow-up. Arch Phys Med Rehabil 2013;94:2068-74.

25. Fryer G. Muscle energy concepts - a need for a change. J Osteopath Med 2000;3:54-9.

26. Burns DK, Wells MR. Gross range of motion in the cervical spine: the effects of osteopathic muscle energy technique in asymptomatic subjects. J Am Osteopath Assoc 2006;106:137-42. 